childhood ages. At age 13 years the HR was 1.12 (95\% CI: 1.01-1.23) per height $\mathrm{z}$-score and HRs were similar across all ages.

Conclusions These findings of positive associations between childhood BMI and height and SLE risk suggest that early life factors may be important in the etiology of SLE.

Funding Source(s): University of Copenhagen Fund for Medical Students and University of Copenhagen, Faculty of Health and Medical Sciences Fund, Gigtforeningen.

\section{ACUTE INFLAMMATORY DEMYELINATING POLYNEUROPATHY AS A PRIMARY MANIFESTATION OF LUPUS IN A PREGNANT PATIENT A RARE AND AN ATYPICAL PRESENTATION}

${ }^{1}$ Anam Umar*, ${ }^{1}$ Muhammad Bilal, ${ }^{2}$ Bina Umar, ${ }^{1}$ Eric Chang, ${ }^{1}$ Balsam Elhammali, ${ }^{1}$ Cinnamon Bradley. ${ }^{1}$ Morehouse School of Medicine; ${ }^{2}$ Henry Ford hospital

\subsection{6/lupus-2019-|sm.117}

Background Systemic lupus erythematosus (SLE) is associated with several neuropsychiatric syndromes involving both the central and peripheral nervous systems. These syndromes are related with SLE progression and thus, are rarely present at the onset of disease. We are reporting a rare case of acute inflammatory demyelinating polyneuropathy (AIDP) as the first manifestation of SLE in a previously asymptomatic patient.

Methods Not applicable as it is a clinical vignette

The abstract for clinical vignette is uploaded as a separate document

Results Not applicable as it is a clinical vignette

The abstract for clinical vignette is uploaded as a separate document

Conclusions Symptoms of AIDP frequently start in lower extremities, yet in about $10 \%$ of patients, arm or facial muscle weakness can be the initial presentation. CSF analysis typically revealed an elevated protein with a normal white cell count; however, a normal CSF protein is observed in onethird to one-half of patients when tested early in the disease course. Our patient had upper extremity weakness as well as initial negative CSF findings, which led to the delay in diagnosis. It is imperative to have a sound knowledge of the typical as well as atypical neurological manifestations of SLE to reduce the morbidity and mortality rate of patients.

Funding Source(s): None

\section{GRANZYME B PRODUCING B CELLS IN SYSTEMIC LUPUS ERYTHEMATOSUS PATIENTS AND LUPUS-LIKE MOUSE MODELS}

Mingxin Bai*, Yin Su. Department of Rheumatology and Immunology Peking University People's Hospital

\subsection{6/lupus-2019-Ism.118}

Background Systemic lupus erythematosus (SLE) is a chronic and systemic autoimmune disease, which is accompanied by abnormal activation of $\mathrm{T} / \mathrm{B}$ lymphocytes, multiple autoantibodies and immune complex deposition. Granzyme B producing $(\mathrm{GrB}+)$ B cells are a group of new regulatory $B$ cell subsets, which can participate in the pathogenesis of autoimmune disease, but its role in SLE is not clear. In this study, we investigate the expression level of Granzyme B producing B cells in SLE patients and lupus-like mouse models.

Methods 1. Patients with SLE $(n=7)$ meeting 1997 American College of Rheumatology revised criteria were enrolled from the Department of Rheumatology and Immunology. 15 healthy individual samples were obtained from physical examination center in our hospital. We collected $4 \mathrm{~mL}$ peripheral anticoagulant blood from both groups and peripheral blood mononuclear cells were isolated. The proportion of $\mathrm{GrB}+\mathrm{B}$ cells in PBMC was identified by flow cytometry. 2. A lupus-like mouse model induced by transfer of spleen cells from bm12 mice was constructed. Two wildtype C57BL/c mice and two lupus-like mouse models (both 68 weeks old mice) were sacrificed by $\mathrm{CO}_{2}$ anesthesia. The spleens of the mice were aseptically isolated, and the spleen single cells were obtained after grinding the tissues. The ratio of $\mathrm{GrB}+$ Breg cells in the spleen cells of the two groups was detected by flow cytometry (FACS). 3. SPSS24.0 software was used for statistical analysis, and $\mathrm{p}<0.05$ was considered statistically significant

Results 1 . Using flow cytometry 7AAD label excludes dead cells, CD3 marker excludes $\mathrm{T}$ lymphocytes and CD56 markers exclude NK cells. After CD14 labeling excludes monocytes/macrophages, the proportion of $\mathrm{GrB}+\mathrm{Breg}$ cells in peripheral blood of healthy controls was 7.52\%; Compared with healthy controls, the proportion of GrB + Breg cells in peripheral blood of SLE patients (4.45\%) showed a significant downward trend. 2. Compared with the wild type control mice, the proportion of $\mathrm{GrB}+$ Breg cells in the spleen cells of lupus mouse model (2.14\%) showed a significant downward trend.

Conclusions The proportion of $\mathrm{GrB}+$ Breg cells in the peripheral blood of SLE patients and the spleen cells of lupus-like mouse models showed a downward trend, which may be related to the pathogenesis of SLE, but the exact role remains to be further verified.

Funding Source(s): This study was supported by grants from the National Natural Science Foundation of China.

\section{NO-SYNTHASE INDUCTIBLE-2 (NOS2) AND VASCULAR ENDOTHELIAL GROWTH FACTOR (VEGF) POLYMORPHISMS IN SYSTEMIC LUPUS ERYTHEMATOSUS AMONG ALGERIAN PATIENTS}

${ }^{1}$ Mounira Benidir*, ${ }^{2}$ Sofiane Samir Salah, ${ }^{3}$ Nesrine Benrebha, ${ }^{4}$ Malik Djennane, ${ }^{5}$ Hachemi Djoudi, ${ }^{6}$ Habiba Amroun, ${ }^{7}$ Ryad Tamouza, ${ }^{8}$ Nabila Attal. ${ }^{1}$ Department of Immunonology, Pasteur Institute of Algeria; ${ }^{2}$ Immunology Department, Mustapha Pacha Hospital; ${ }^{3}$ Medical Biology Department, Blida Hospital; ${ }^{4}$ Rheumatology Department, TiziOuzou Hospital; ${ }^{5}$ Rheumatology Department, Douera Hospital; ${ }^{6}$ Medical Biology Department, Hussein Dey Hospital; ${ }^{7}$ Department of Immunology and Immunogenetics, Henri Mondor Hospital, Paris. France; ${ }^{8}$ Immunology Department, Pasteur Institute of Algeria

\subsection{6/lupus-2019-Ism.119}

Background The development of Systemic Lupus Erythematosus (SLE) depends inter alia on genetic factors including genes involved in oxidative stress and angiogenesis as NOS2 and VEGF. The aim of our study is to evaluate the Single Nucleotide Polymorphisms (SNPs) influence of NOS2 gene (rs2779248, rs2779251 and rs8078340) and VEGF gene (rs1570360 and rs2010963) on SLE development in Algerian patients.

Methods This is a case-control study of 157 SLE patients (age: $37 \pm 2$ years, sex ratio: 1: 10, disease duration: $7.6 \pm 4.3$ years, SLEDAI: $7.3 \pm 6.1$ ) and 173 healthy controls (age: $28 \pm 9$ years, sex ratio: 1: 7). We performed NOS2 and VEGF genes 